\title{
Applicabilité et utilité de la typologie de Larouche et al. (2012) comme outil de gouvernance, de planification et de gestion : le cas de l'Université de Moncton
}

\author{
Catherine Larouche ${ }^{a}$, Denis Savard ${ }^{b}$, Jean-François Richard ${ }^{c}$, Gabrièle Leblanc $^{d}$, \\ Pauline Jean ${ }^{\mathrm{e}}$
}

RÉSUMÉ. La typologie des conceptions des universités de Larouche et al. (2012) a été développée et validée en vue d'évaluer la performance des établissements universitaires. Cette typologie comporte sept types de conceptions (Académique, Service public, Marché, Entrepreneuriale, Politique, Milieu de vie et Apprenante). De façon à juger de l'applicabilité de la typologie dans les processus de gouvernance, de planification et de gestion des établissements universitaires, huit administrateurs de l'Université de Moncton ont été rencontrés dans le cadre d'entretiens semi-structurés. Cette communication a comme objectif de rendre compte, d'une part, de la perception des dirigeants rencontrés par l'identification des conceptions présentes et de réalités signifiantes à cette université, et d'autre part, de l'utilité de la typologie comme outil de gouvernance, de planification, d'évaluation ou de gestion.

ABSTRACT. The typology of conceptions of Universities of Larouche et al. (2012) was developed and validated to assess the performance of academic institutions. This typology comprises seven types of conceptions (Academic, Public Service, Market, Entrepreneurial, Political, Living Environment, and Learning Organization). Eight administrators from Moncton University were met during semi-structured interviews to judge the typology's applicability in the governance, planning, and management processes of academic institutions. The purpose of this communication is to report, on the one hand, on the perception of the leaders encountered through the identification of present concepts and significant realities at this university, and on the other hand, on the typology's usefulness as a tool for governance, planning, evaluation or management.

\section{Introduction}

Au début des années 2000, les universités québécoises ont été soumises à des contrats de performance comportant des objectifs chiffrés mesurés par des indicateurs quantitatifs. Cette opération a été l'occasion d'une réflexion sur la performance des universités (Larouche, 2011), celles-ci ne pouvant être réduites à une vision mécanique d'une entreprise qui possède une hiérarchie bien campée, des processus de production récurrents et des tâches planifiées avec exactitude (Amaral, Meek et Larsen, 2003; Axelrod, 2002; Bruckmann et Carvalho, 2014), ni à un ensemble réduit d'indicateurs quantitatifs. Au-delà de leur triple mission (enseignement, recherche et service à la collectivité), le bon fonctionnement des établissements universitaires repose sur l'agencement complexe des valeurs, des objectifs et des actions parfois antagonistes d'une multitude d'acteurs, autant internes qu'externes, le tout couvrant un large spectre disciplinaire. Birnbaum (1988)

\footnotetext{
a Professeure, Université du Québec à Chicoutimi

b Professeur titulaire, Université Laval

c Vice-recteur adjoint à l'enseignement et aux affaires professorales, Université de Moncton

d Étudiante à la maîtrise, Université du Québec à Chicoutimi

e Étudiante au doctorat, Université Laval
} 
estime que pour examiner la complexité des universités, il convient de clarifier les objectifs conflictuels et redéfinir les pratiques de gestion et les modèles d'évaluation en fonction de cette complexité. Or, c'est, entre autres, à ce problème que s'adresse l'approche typologique adoptée par Larouche, Savard, Héon et Moisset (2012).

La typologie des conceptions des universités de Larouche et al. (2012) a été développée et validée en vue d'évaluer la performance des établissements universitaires. Cette typologie comporte sept types (Académique, Service public, Marché, Entrepreneuriale, Politique, Milieu de vie et Apprenante) définis selon sept dimensions (valeurs et principes, système de gouvernance, stratégies, enseignement, recherche, modes d'évaluation et critères d'évaluation) ${ }^{1}$.

Une subvention provenant du Programme de coopération intergouvernemental (PCI) a été obtenue en 2016 et renouvelée en $2017^{2}$, dans le but de favoriser la coopération entre les chercheurs de l'Université du Québec à Chicoutimi et de l'Université de Moncton. Une première subvention PCI-2016 a permis de vérifier l'applicabilité de la typologie de Larouche et al. (2012) dans le contexte d'une université francophone de l'Est canadien, soit l'Université de Moncton, et d'explorer auprès des dirigeants de cette université son utilité comme outil de gouvernance, de planification, d'évaluation ou de gestion. Les dirigeants rencontrés sont d'avis qu'il s'agit d'un outil de réflexion qui permet la clarification des enjeux et qui pourrait être utile dans les opérations de gestion, de planification et d'évaluation des universités. Cette communication a donc comme objectif de rendre compte de la perception des dirigeants rencontrés dans le cadre de ce projet de recherche qui visait les objectifs suivants : 1) vérifier l'application de la typologie de Larouche et al. (2012) dans le contexte d'une université francophone d'une province de l'Atlantique; 2) explorer auprès des dirigeants de cette université l'utilité de la typologie comme outil de gouvernance, de planification, d'évaluation ou de gestion.

Cette communication comporte trois parties. Une première partie présente la typologie des conceptions des universités visant à évaluer leur performance (Larouche et al., 2012); dans la deuxième partie, la méthodologie de cette étude est brièvement décrite et enfin, dans la troisième partie, les résultats des entrevues avec les dirigeants de l'Université de Moncton sont exposés.

\section{La typologie}

La typologie de Larouche et al. est conçue spécifiquement pour rendre compte de la complexité et de la spécificité des universités dans l'évaluation de leur performance. Cette typologie repose sur le postulat qu'au-delà de la mission fondamentale des universités coexistent en leur sein une pluralité de conceptions qui teintent cette mission. Ces conceptions varient dans le temps, dans l'espace, selon des rythmes d'alternance et de prédominance, et en fonction des instances et des individus. Cette typologie s'inspire principalement des travaux de Mintzberg, Ahlstrand et Lampel (1999), de Morgan (1999) et de Morin, Savoie et Beaudin (1994). La typologie est décrite succinctement ci-dessous et de façon plus étendue dans le tableau $1^{3}$ :

- Dans la conception Académique, l'Université porte et exprime certaines finalités idéales qui transcendent la temporalité et l'espace (Freitag, 2002). L'Université ${ }^{4}$ concrétise une idée civilisatrice qui vise la formation des citoyens à un idéal d'humanité, de culture et de vie commune (Freitag, 2002). On y recherche la vérité, on valorise le développement du savoir et la rigueur scientifique. Au sein de cette conception, la liberté académique s'avère une valeur fondamentale (Larouche et al., 2012).

- Pour la conception Service public, l'éducation, l'enseignement supérieur et la formation universitaire constituent un bien personnel et social (MEQ, 2000). On conçoit l'Université au service de tous, comme un bien commun faisant partie du patrimoine collectif (FQPPU, 1997). L’Université voit à la réussite et à la qualification. Elle est ouverte sur la société : elle se montre capable de répondre à ses attentes et de contribuer au progrès de la collectivité dans son ensemble (Larouche et al., 2012).

- La conception Marché décrit une Université ayant une valeur marchande (Porter, 1993). Elle est soumise aux lois d'un marché autorégulateur. La logique de marché se présente à 
travers l'idée de capacité à développer l'innovation afin de répondre à une demande déterminée dans un contexte de concurrence régionale, nationale et internationale. L'idée de marché s'articule principalement autour des questions de positionnement concurrentiel et de satisfaction de la clientèle, autant sur le plan de l'enseignement que dans ceux de la recherche et des services à la collectivité (Larouche et al., 2012).

- La conception Entrepreneuriale vise à exploiter les ressources et les possibilités de développement au-delà des moyens disponibles (Clark, 1998). L'établissement s'adapte à un univers complexe et incertain, mais le fait à ses propres conditions. Cette conception se distingue de la conception de marché dans la mesure où elle repose sur la logique de l'offre, marquée par le goût du risque, la créativité, le développement de productions novatrices, alors que la conception de marché concerne la demande et se traduit par des productions adaptées aux besoins identifiés (Larouche et al., 2012).

- Les tenants de la conception Politique recherchent la légitimité auprès des parties prenantes (Morin, Savoie et Beaudin, 1994;
Quinn et Rorhbaugh, 1983). Il s'agit ici d'obtenir le soutien et la confiance des acteurs de son environnement. Les tenants de la conception Politique mettent l'accent sur le changement social en luttant contre les forces dominantes et en cherchant l'équité entre les différentes composantes de la société (Larouche et al., 2012).

- La conception Apprenante s'inspire du concept d'organisation apprenante (Duke, 1992; James, 2000; Mead, 1995; Ramsden et Martin, 1996). L'organisation apprenante crée, acquiert, transfère des connaissances et modifie sa conduite pour refléter des connaissances et des perspectives nouvelles (Dill, 2003; Garvin, 1993; Larouche et al., 2012).

- La conception Milieu de vie prône que la pleine réalisation des capacités de l'être humain implique la connaissance de soi, la liberté d'agir sur sa propre destinée, la légitimité de la recherche du bonheur, la tolérance des idées diverses et un idéal commun de justice. Cette interprétation s'appuie sur un partage des traditions, des valeurs et des habitudes propres à la vie de l'organisation (Mintzberg, Ahlstrand et Lampel, 1999). Cette conception suppose un fort sentiment d'appartenance (Larouche et al., 2012). 


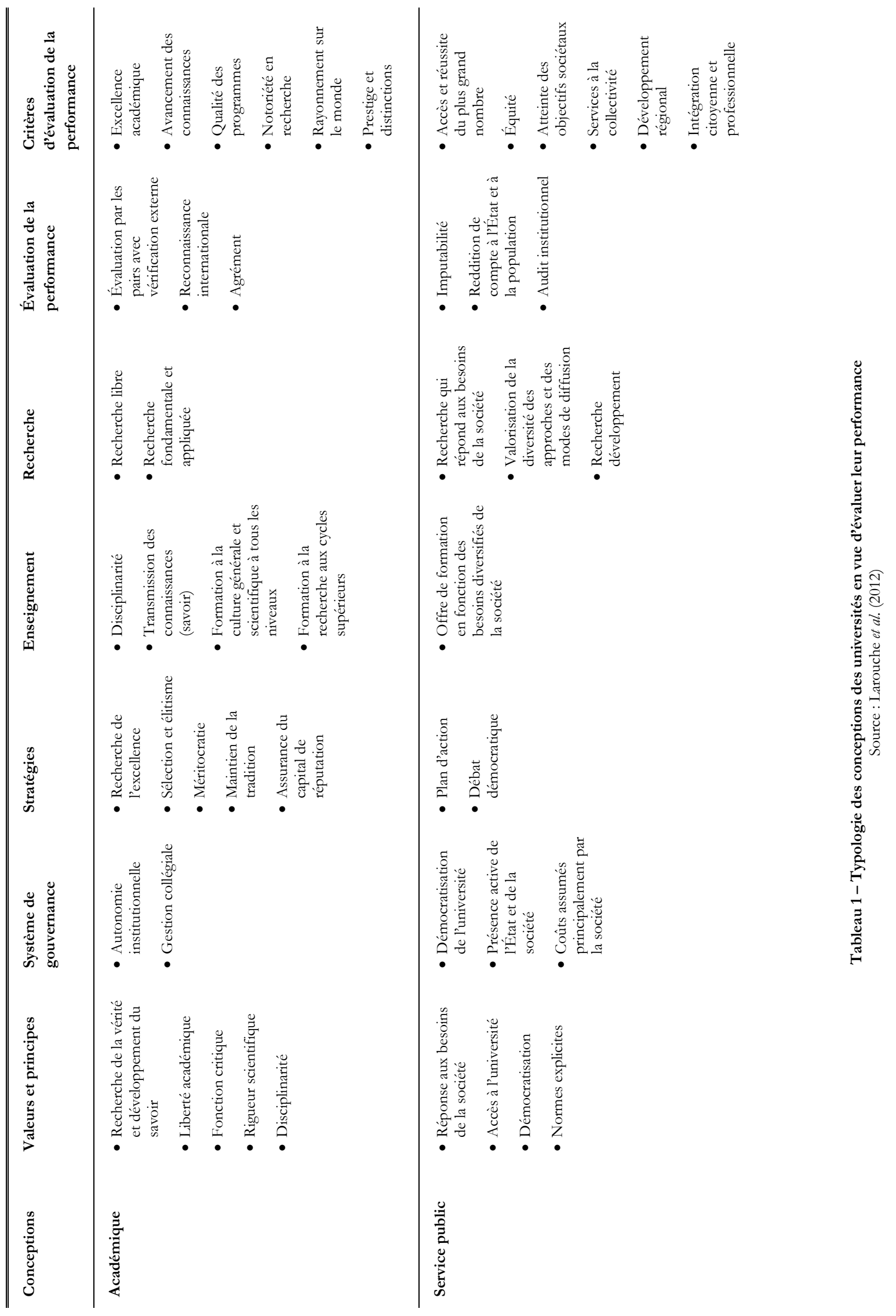




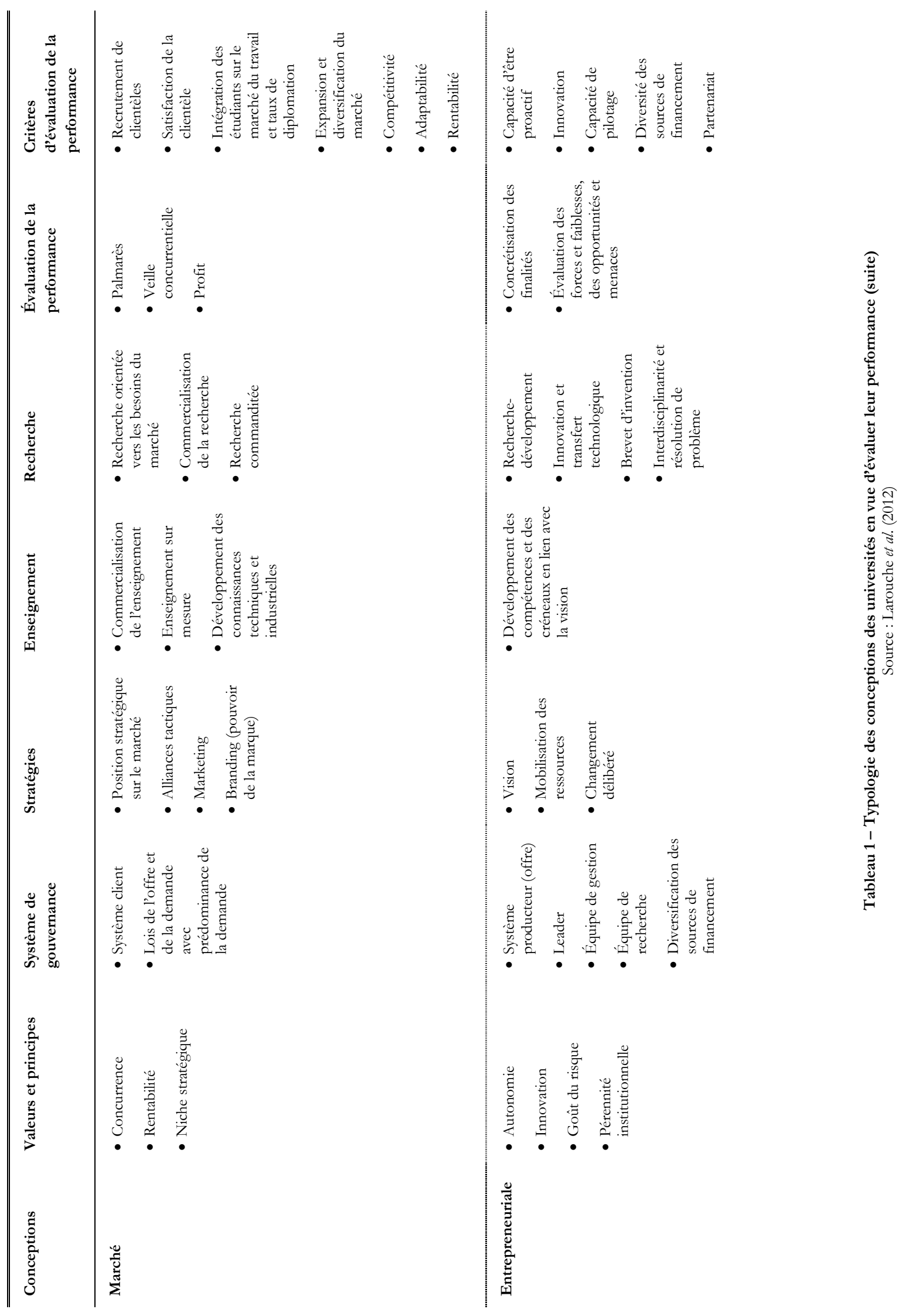




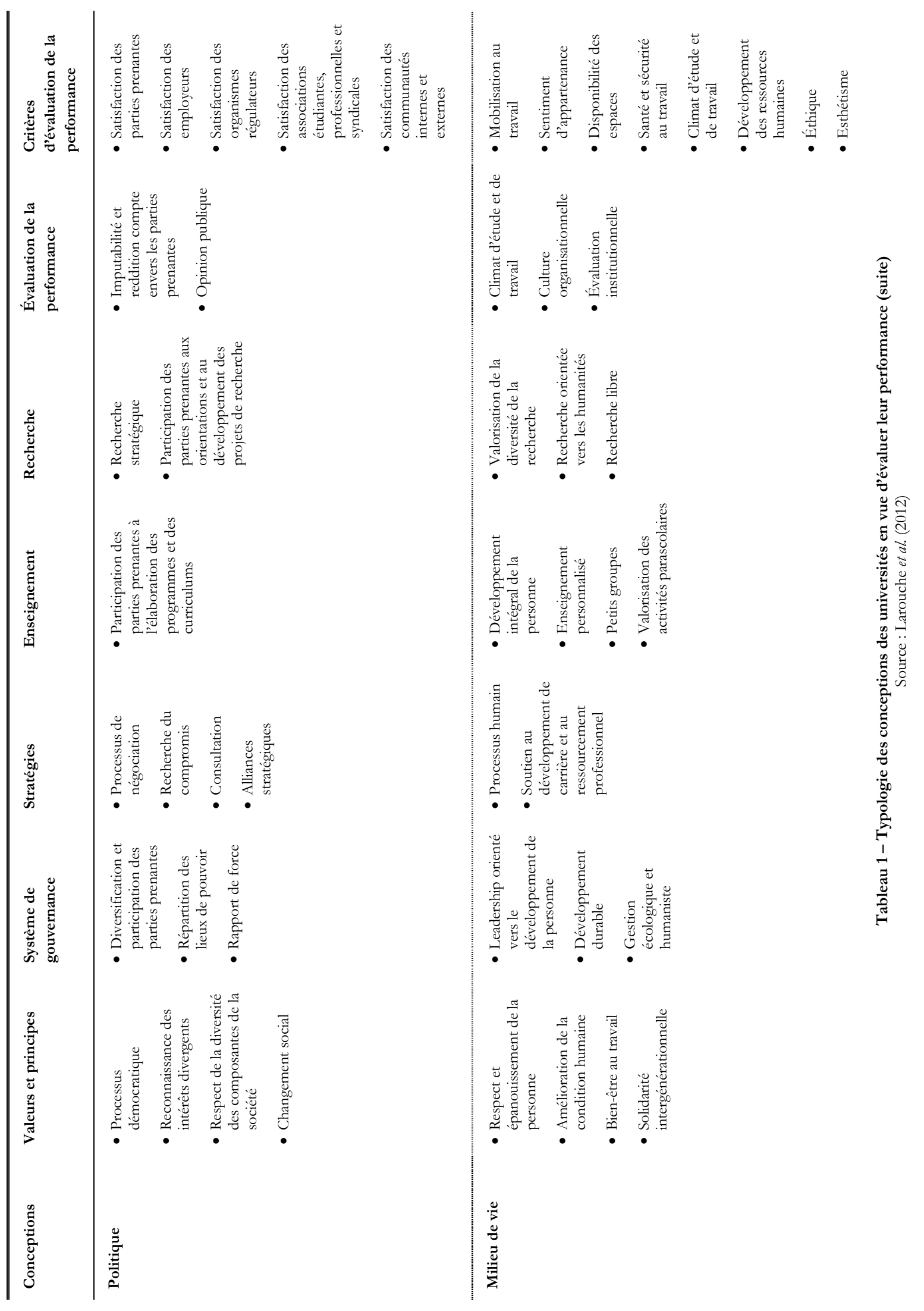




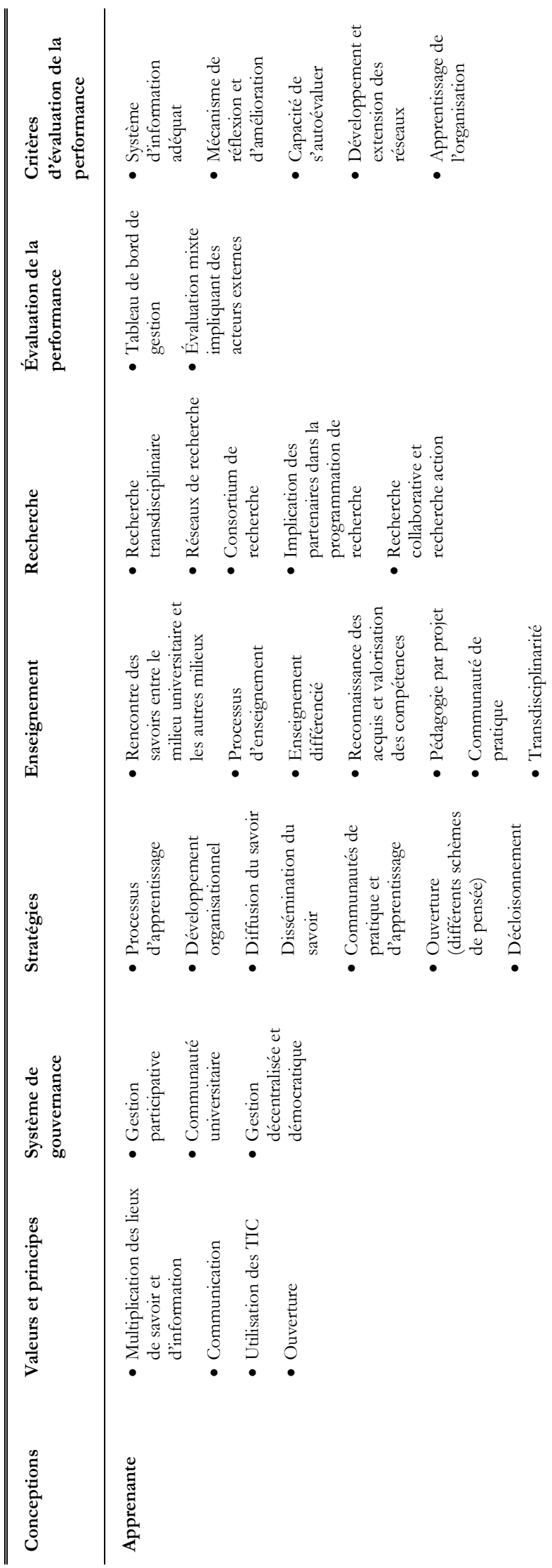




\section{La méthodologie}

De manière à juger de l'applicabilité de la typologie dans les opérations de gouvernance, de planification stratégique, d'évaluation et de gestion, huit administrateurs (vice-recteurs ou doyens ${ }^{5}$ ) de l'Université de Moncton ont été rencontrés dans le cadre d'entretiens semi-structurés' ${ }^{6}$ Le schéma d'entretien comportait huit questions. Les entretiens se sont déroulés entre les 14 et 21 décembre 2016. Sept entretiens se sont déroulés à l'Université de Moncton et un entretien a été réalisé par appel téléphonique. La durée moyenne des entrevues est de 38 minutes.

\section{Les résultats}

Les résultats sont présentés en cinq parties. La première partie concerne la perception des dirigeants interrogés sur la typologie dans sa globalité. La deuxième partie présente la diversité des conceptions de l'Université de Moncton selon les perceptions des dirigeants. En troisième partie, les réalités signifiantes mentionnées par les dirigeants sont décrites. La quatrième partie aborde l'utilité de la typologie comme outil de gouvernance, de planification, de gestion et d'évaluation et distingue les acteurs susceptibles de l'utiliser selon les perceptions des dirigeants. Enfin, la cinquième partie identifie des conceptions qui devraient prendre plus d'importance dans le futur selon les dirigeants.

\subsection{Perception globale de la typologie}

Tous les participants estiment que la typologie est intéressante à plusieurs égards. Elle révèle les conceptions de la société civile ou des acteurs de l'enseignement supérieur par rapport au rôle de l'université. Les participants ont relevé plusieurs réalités signifiantes associées à chacune des conceptions ${ }^{7}$. La typologie reflète l'université dans beaucoup de ses activités (E1) ${ }^{8}$. On note qu'une université peut s'identifier à plus d'une conception et même, dans certains cas, toucher à l'ensemble des conceptions (E1 et E3). Le fait que la typologie autorise un amalgame de deux ou trois conceptions dominantes pour l'identification d'une université la rend intéressante (E8).

Des liens sont établis entre les différentes conceptions : certaines se recoupent (E6), se complètent, ou sont en opposition (E7), par exemple, en ce qui concerne la nature de la recherche comme le fait de produire des résultats de recherche utilisables (p. ex. : pour la commercialisation) en opposition à faire avancer un savoir disciplinaire (E7).

La typologie donne une vision de ce qu'est et doit être l'université (E6). Elle permet de mettre l'accent sur ce qui est important (E2), de clarifier sa propre réflexion (E5) et de décortiquer les différentes conceptions que les gens peuvent avoir des universités (E8).

La typologie favorise la réflexion et suscite le questionnement (E4). Certaines conceptions (Marché et Entrepreneuriale), par contre, soulèvent des questions. Pour certains, la conception Marché est vue comme externe à l'université, imposée par le gouvernement et, pour d'autres, elle apparait comme une réalité avec laquelle il faut composer pour assurer la rentabilité.

Les participants donnent plusieurs exemples de l'utilité de la typologie comme outil de gouvernance, de gestion ou d'évaluation9. Tous affirment son utilité dans le processus de planification stratégique. Les critères de mesures de la performance sont vus comme intéressants pour assurer l'évaluation subséquente à la planification (E5).

\subsection{La diversité des conceptions de l'Université de Moncton selon les perceptions des dirigeants}

Les propos des dirigeants interrogés sur chacune des conceptions sont rapportés ainsi que l'identification de leur conception dominante.

\section{La conception Académique}

La conception Académique est considérée comme une conception dominante de l'Université de Moncton par E1, E2, E3, E4, E5, E6, E7, E8. La conception Académique, la transmission du savoir, la construction du savoir au sein de la société acadienne, c'est extrêmement important dans la conception que l'on a du rôle de l'Université de Moncton (E7). La conception Académique se trouve aux fondements de l'Université (E1, E2). Cette conception fait référence à la recherche de la vérité, au développement des connaissances, à la fonction critique et à la liberté académique; plusieurs secteurs de l'université s'identifient à cette conception (E3, E7). La conception Académique comporte parfois une part d'opposition avec la conception Service public, car il faut, comme seule université francophone de cette province, offrir beaucoup de 
programmes dans une université de taille plutôt modeste (E8).

La gestion collégiale caractérise la conception Académique. E6 mentionne que la gestion collégiale, quoique souvent évoquée à l'université, est très peu mise en pratique. Ce fait est probablement lié au contexte qui est difficile en matière de ressources financières qui pousse les administrateurs à se replier sur soi et à perdre cette notion de collégialité (E6).

\section{La conception Service public}

La conception Service public est identifiée comme une conception dominante de l'Université de Moncton par E1, E3, E4, E6, E7, E8. La mission de cette université est historique, elle a été créée par et pour l'Acadie (E1, E4). Depuis sa création, il y a 50 ans, l'Université de Moncton a été le vecteur du développement de la société acadienne (E8). L'accès à l'Université, surtout pour le peuple acadien, constitue un élément crucial de l'histoire de l'Université de Moncton (E6, E8). On est passé d'une diplomation universitaire très faible à très élevée. (E8).

Le premier mandat de l'Université de Moncton consiste à offrir une diversité de programmes et de formations en français en vue de répondre aux besoins de la société acadienne (E2, E4 et E8). Son rôle en tant que service public est important puisque l'Université de Moncton répond aux besoins de la communauté acadienne (E1, E6, E7), elle se préoccupe de son développement et de son rayonnement (E1, E2, E4).

Selon E2, la conception Service public était plus importante il y a 50 ans qu'elle ne l'est aujourd'hui. On voit les universités de moins en moins comme un service public, davantage comme un service parapublic ou privé (E2). Pour E6, la démocratisation et l'accès à l'Université demeurent aujourd'hui plus importants que les dimensions de concurrence, de rentabilité ou de niche stratégique (E7).

\section{La conception Milieu de vie}

La conception Milieu de vie est considérée comme dominante par E3, E5, E7 et E8. Depuis un certain nombre d'années, la conception Milieu de vie prend davantage de place à l'université (E7). On se soucie du respect entre les individus, on met en place des politiques qui protègent les personnes contre le sexisme, le harcèlement, etc. (E7).
La conception Milieu de vie s'avère une conception importante parce qu'elle reflète le souci du développement et de l'épanouissement de la personne (E5, E4). Cette conception est associée aux besoins des étudiants, notamment en ce qui a trait à la santé mentale. Les étudiants sollicitent un milieu de vie mieux adapté et répondant adéquatement à leurs besoins. Cet intérêt pour le bien-être des étudiants représente un changement de culture (E5, E4).

De l'avis de E6, on dénombre plusieurs problématiques (santé mentale, relations de travail, etc.) pour lesquelles les gestionnaires ne sont pas tous bien outillés. Il n'est peut-être donc pas possible d'associer l'Université de Moncton à la conception Milieu de vie (E4) quoique la part accordée à cette conception ait augmenté. Cet objectif devrait faire partie de la planification stratégique, ce qui n'est pas le cas présentement (E4, E6). Un lien très fort existe entre l'université et la communauté qu'elle dessert (E4). D'ailleurs, l'Université de Moncton, selon E7, est issue d'une certaine conception de la communauté qui s'inscrit dans une tradition humaniste de responsabilité citoyenne. Enfin, précise E4 : «L'Université de Moncton se trouve au cœur de l'Acadie ».

\section{La conception Apprenante}

La conception Apprenante est considérée comme dominante par E2 et E7. La conception Apprenante et l'utilisation des TIC sont présentes à l'Université de Moncton (E5). L'aspect du partenariat avec d'autres universités fait également partie des possibilités qu'ont amenées les TIC (E5). L'université est de plus en plus sensible aux éléments associés à cette conception principalement avec la formation à distance et l'éducation permanente (E1, E6), mais pour l'instant, cela n'est pas tout à fait atteint (E6). Selon E3, à l'exception de l'éducation permanente, il existe beaucoup de résistance de la part du corps professoral. Cette nouvelle vision de l'ingénierie pédagogique et l'utilisation des TIC font peur aux professeurs qui préfèrent un enseignement plus traditionnel (magistral) (E3).

\section{La conception Entrepreneuriale}

La conception Entrepreneuriale est identifiée comme dominante par E2. En ce qui concerne cette conception, il faut être conscient que les universités sont conservatrices et peu enclines à l'innovation et au changement (E5). Pour assurer la pérennité, il faut prendre des risques et bouger vite, choses plus diffi- 
ciles pour les universitaires qui sont souvent des personnes hyper perfectionnistes. Pour cette raison, la conception Entrepreneuriale n'est pas là, mais il est nécessaire de la faire émerger (E5). E2 est d'avis que la vision même de ce qu'est l'Université a changé; elle doit s'adapter aux changements vécus dans la société. Elle doit répondre aux besoins de sa clientèle, de la société, du gouvernement (E2). De plus en plus, elle doit développer de nouvelles technologies et de nouveaux partenariats avec l'industrie (E2). Étant donné le contexte de forte diminution des effectifs et du financement, il faut innover (E8). Cependant, E6 précise que limiter l'Université à être une entreprise risque de dénaturer sa raison d'être.

\section{La conception Marché}

Cette conception n'est pas considérée comme une conception dominante. Certains la considèrent comme une conception extérieure à l'université (E2). E2 insiste sur le fait qu'aujourd'hui les universités se cherchent, changent et doivent s'adapter. Pour cette raison, la conception Marché s'avère de plus en plus présente (E2), mais ne serait pas dominante (E3). Cette situation s'explique par le contexte financier difficile (E4).

Beaucoup de programmes de l'Université de Moncton sont axés sur des spécificités régionales (p. ex. : Gestion des zones côtières). Un positionnement stratégique permet de se démarquer par rapport à la concurrence (E1). E5 est d'avis que la satisfaction de l'effectif (qui est vu comme une clientèle dans cette conception) est de plus en plus prise en compte et importante. L'université doit recruter des étudiants, elle est en compétition avec d'autres universités et elle doit également répondre à la demande du marché, mais sans toutefois s'y soumettre au risque de se dénaturer (E6).

\section{La conception Politique}

La conception Politique n'est pas considérée comme une conception dominante. La conception Politique (volonté de changement social) est présente, car l'Université de Moncton a été créée, comme l'indique sa mission, pour promouvoir la communauté acadienne (E3). Selon E6, on n'a pas le choix car on se retrouve toujours dans des rapports de force. Même si E6 n'est pas convaincu que la conception Politique définit vraiment l'Université de Moncton, il est d'avis que l'on pourrait faire davantage en cette matière. En raison des relations politiques et de la baisse de financement, il est difficile de jouer pleinement ce rôle (E6 et E4). Le fait que certains professeurs soient critiques et très actifs dans les médias n'est pas toujours apprécié par les gestionnaires; cela dérange (E6). Cette conception n'est pas, de façon générale, primordiale à l'Université de Moncton(E7), mais elle pourrait s'avérer importante pour des gens provenant de certaines disciplines (E2).

Le schéma qui suit illustre les conceptions mentionnées comme dominantes à l'Université de Moncton par les participants.

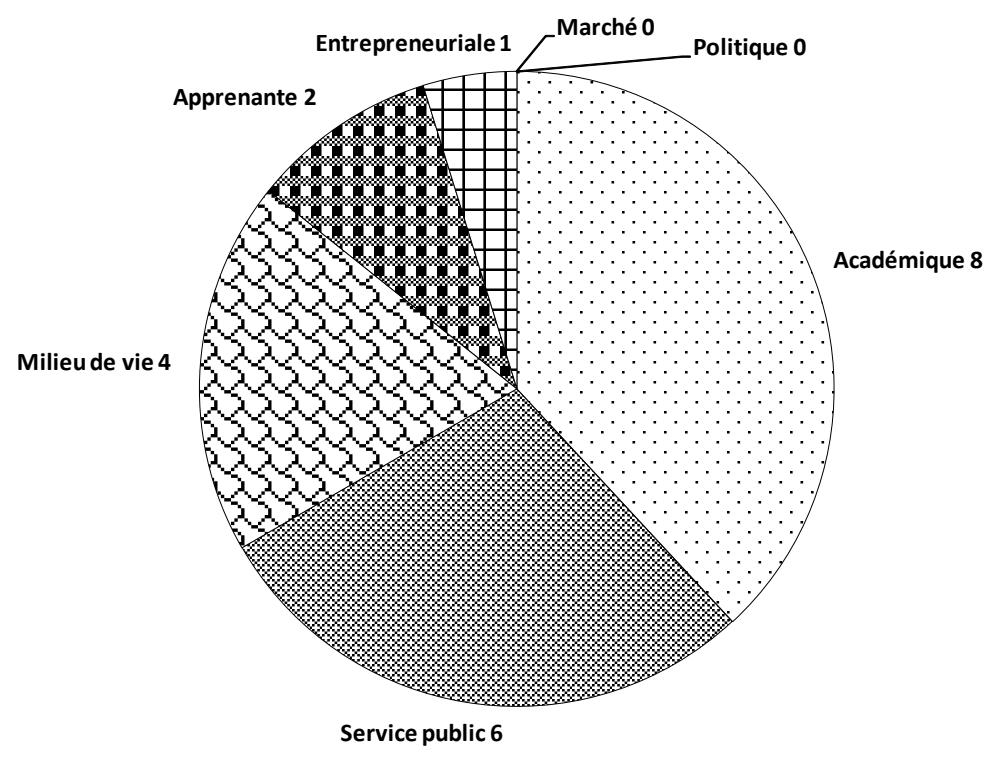

Figure 1 - Les conceptions dominantes de l’Université de Moncton mentionnées par les participants 


\subsection{Les réalités signifiantes}

Si les conceptions varient d'une faculté à l'autre, mais également d'un individu à l'autre, elles peuvent également varier selon les moments, les contextes et même en fonction des dirigeants en place (E6).

\section{Selon les disciplines ou les facultés}

Certaines conceptions sont des concepts parapluie rejoignant ou touchant tout le monde, alors que d'autres concernent plus spécifiquement certaines disciplines ou facultés (E4). À l'Université de Moncton, comme plus de 18 disciplines y sont enseignées, il y a évidemment des divergences quant aux points de vue et visions de ce que doit être l'Université. Certaines tensions existent entre les disciplines, les facultés. De très grands défis se profilent pour rallier tout ce monde autour d'un projet commun (E3). Plusieurs unités d'enseignement et administratives adoptent des conceptions différentes, cela fait partie de la dynamique et de la réalité de l'université (E4).

Les conceptions s'associent davantage aux unités qu'à l'université dans son ensemble. Par exemple, les conceptions Marché et Entrepreneuriale, s'adapteraient peutêtre davantage aux disciplines professionnelles que fondamentales (E4). Certaines facultés privilégient la conception Milieu de vie alors que d'autres optent pour la conception Entrepreneuriale ou Académique (E4).

La Faculté de génie, la Faculté des sciences (E4, E1), celles des arts et des sciences sociales (E6) peuvent être associées à la conception Académique (E8). En raison du développement de brevets et d'innovations (E8), la conception Entrepreneuriale peut aussi être associée à la Faculté de génie et à la Faculté des sciences.

Les sciences infirmières et les sciences de l'éducation auraient un lien plus fort avec la conception Service public parce qu'elles forment des professionnels devant travailler directement dans un système public qui en a besoin (E2, E8). Les conceptions Académique (E8), Apprenante et Entrepreneuriale seraient aussi présentes dans le secteur de l'éducation (E6, E4).

\section{Selon les acteurs}

Les réalités diffèrent selon les observateurs (E8). Il sera difficile d'arriver à un consensus sur les conceptions dominantes au sein d'une même université. D'ailleurs, les différences entre les individus expliquent les difficultés à mettre en œuvre une politique ou un changement (E8).

Les professeurs expriment des points de vue très différents : certains se disent ouverts à travailler avec l'industrie, alors que d'autres ne le souhaitent pas (E2). À l'intérieur d'une même faculté, il faut être conscient que des individus prônent la conception Académique et refusent la conception Politique: ces deux modèles s'opposent (E3). Certains aimeraient que la conception Apprenante prenne plus de place. La conception Entrepreneuriale n'est pas une conception très populaire (E3).

Le corps professoral adopterait davantage une conception Académique de l'université, à travers la liberté académique, l'excellence, la recherche fondamentale, alors que les administrateurs auraient une perspective différente en raison des budgets et des demandes de la société (E8, E6). Pour certains professeurs, les conceptions Entrepreneuriale on Marché ne passent pas du tout (E8).

\subsection{L'utilité de la typologie}

La typologie peut être utile pour la planification, la gouvernance, la gestion et aussi pour l'évaluation (E2).

\section{Outil de gouvernance et de planification}

Les répondants trouvent une utilité à la typologie pour la planification stratégique (E1, E2, E4, E6, E7). La typologie apporterait un éclairage complémentaire à l'exercice réalisé récemment à l'Université de Moncton pour 2020 (E5). Son utilisation permettrait de peaufiner le travail effectué pour qu'il réponde davantage à la vision de ce que l'on souhaite adopter (E5). $\mathrm{Au}$ moment de la rencontre d'évaluation de fin d'année alors que s'effectue le bilan des actions réalisées, il serait intéressant de faire l'exercice en équipe de direction en mettant à profit la typologie (E5). Cette dernière permet d'identifier des valeurs communes aux différentes facultés; ce qui pourrait s'avérer fort pertinent, surtout dans une période de transition. Une partie du corps professoral n'est pas prête aux changements prévus pour 2020, et la réalité d'aujourd'hui, sur les plans du recrutement, du financement et de l'accès nécessite de se renouveler pour être compétitif et offrir des programmes de qualité. Réussir à faire plus avec moins, c'est un réel défi pour l'Université de Moncton (E5). 
La typologie s'avère utile durant la réalisation de la planification prévisionnelle et stratégique des effectifs. Cela devrait permettre de passer d'un cadre plus théorique, centré davantage sur une vision académique, à une vision plus pratico-pratique, basée sur l'action (E5). Enfin, l'utilité de la typologie peut se révéler dans la planification académique des facultés, présentement en voie de réalisation (E2).

\section{Outil de réflexion pour élaborer une vision et des orientations (analyse des forces et faiblesses)}

La typologie permet aux institutions de mieux se connaître, de se définir et d'orienter leur devenir (E4, E6, E7). Elle amène une vision plus claire des objectifs poursuivis (E4, E3). Elle pose des mots sur des réalités (E3) et des définitions sur les idées (E2). Elle associe les valeurs, les styles de gouvernance, les mesures de performance aux objectifs et aux orientations visées selon E3 qui aimerait utiliser la typologie dans le cadre de ses fonctions.

La typologie fait ressortir les éléments importants et névralgiques (E5), que ce soit à l'interne ou à l'externe (E2) pour mieux se situer (E5). Par exemple, à la suite d'une série de questionnaires auprès de différents acteurs, il est possible de faire des liens entre les résultats obtenus et les éléments constituant la typologie. On peut ainsi prendre conscience des conceptions dominantes, celles qui sont en émergence et identifier des tensions et des différences d'opinions entre les acteurs (E1). Un autre exemple est donné en ce qui concerne le nombre de choix de cours qui diminue pour permettre une économie budgétaire. Ceci produit un effet indéniable sur la gestion de la réputation de l'université et ultérieurement sur le recrutement (E5). Mais il faut que cela soit présenté dans le cadre d'un échange sur le sujet à l'occasion d'une planification ou d'une évaluation (E5).

\section{Outil de consensus}

La typologie favorise la compréhension mutuelle et l'atteinte de consensus sur les plans de la gouvernance, de la gestion et de l'évaluation (E7).

\section{Outil d'analyse critique}

La typologie ouvre vers une perspective critique des rapports et des discours relatifs à la gouvernance et à la gestion de l'université (E2).

\section{Outil de gestion}

La typologie aide la gestion d'une faculté (embauche de personnes, prise de décision, orientation et justification) (E2, E1, E6).

\section{Outil d'évaluation}

La typologie sert à l'évaluation (E1, E6). Par exemple, l'évaluation des professeurs ou des recherches est souvent réalisée de manière traditionnelle et académique (E1). Dans le cas de professeurs travaillant avec l'industrie et ayant développé des façons de faire distinctes ou des projets expérimentaux innovants, cette évaluation dite «traditionnelle» n'est souvent pas appropriée. La typologie offre un outil précieux pour l'évaluateur: elle autorise une évaluation qui tient davantage compte des spécificités. La typologie apporte donc une vision plus juste, plus claire de la réalité $(\mathrm{E} 1)$.

La typologie peut être utilisée pour vérifier l'atteinte des objectifs (E4). Elle améliore la capacité d'autoévaluation (E6), d'évaluation de certaines stratégies en vue de les associer aux besoins de l'université (E7). L'outil fournit l'occasion de mesurer les différentes façons de concevoir l'université et d'éviter de faire des évaluations partielles comme c'est le cas présentement (E8). Il serait intéressant de rectifier l'image voulant que l'Université ne soit pas le vrai monde; l'Université est le vrai monde et répond à des besoins qui sont extrêmement vastes (E8).

\subsection{Les personnes susceptibles d'utiliser la typologie}

Les administrateurs, les professeurs, les instances de tutelle ou de gestion, le gouvernement peuvent trouver de l'intérêt à l'utilisation de la typologie.

\section{Les administrateurs}

Les administrateurs sont mentionnés en premier lieu, comme usagers potentiels de la typologie, car ils emploient constamment les concepts qu'elle renferme (E2, E3, E6, E8). Cela est important pour l'équipe de direction qui doit prendre des décisions administratives (E3, E4, E5).

Les professeurs

Les professeurs ne seront pas nécessairement intéressés par ce genre d'outil (E1, E3, E4, E6, E7), quoique E2 soit d'avis contraire. Ce serait bien, mais plutôt 
utopique, d'impliquer les professeurs : ils sont dans une bulle, ailleurs (E5). Peut-être serait-il pertinent de trouver une façon de les impliquer en approchant et en impliquant des instances comme l'ACPU (Association canadienne des professeurs universitaires)? Par exemple, la typologie pourrait être présentée à certains groupes d'enseignants afin de les sensibiliser et même les former (E5).

\section{Le gouvernement et la société civile}

Le gouvernement pourrait être intéressé par la typologie (E1, E2, E3, E4) de même que la société civile (E6). Cela permettrait de prendre conscience de la spécificité et de la nature diversifiée de l'Université (E6 et E4) et faciliterait les échanges, le dialogue et les négociations entre le gouvernement et les universités (E6) : il faut comprendre la façon dont fonctionne l'université pour prendre des décisions éclairées. Les politiques universitaires sont souvent décidées sur la base de critères ponctuels et à court terme, sans vision à long terme. Si les preneurs de décision et le gouvernement étaient conscients de ces conceptions, peut-être feraient-ils preuve de plus de sagesse dans leurs décisions (E4). Le gouvernement se dirige plutôt vers l'utilisation d'indicateurs de performance uniformisés. Une lecture de la typologie permettrait aux acteurs des instances gouvernementales d'avoir une vision plus juste du rôle de l'université (E6) avant de proposer ces indicateurs.

Il importe que les universités développent des niches, des programmes mieux adaptés aux besoins du milieu, et ce, en complémentarité avec les autres institutions universitaires; la typologie peut servir à réfléchir à ces questions (E6). Enfin, pour E5, le gouvernement ne peut utiliser la typologie puisque sa vision est trop «collège communautaire » et directement axée sur l'emploi.

\subsection{Les conceptions en émergence}

Les conceptions Académique (E2 et E6), Apprenante (E2) et Service public (E4, E6) devraient toujours être présentes. Cependant, comme le précise $\mathrm{E} 4$, cela va être difficile pour le système universitaire canadien de maintenir une conception Académique dominante dans les années à venir à cause des pressions énormes de l'extérieur. C'est de plus en plus difficile de gérer les universités et de les financer, de demeurer des uni- versités avec un grand U, comme on l'a conçu pendant des décennies; c'est-à-dire de voir l'Université comme le temple du savoir et de la recherche pure, de maintenir la liberté académique (E4).

Les conceptions Entrepreneuriale et Marché devraient prendre plus de place dans le futur (E4, E2, E6 et E8) en raison des changements vécus par la société. L'université devra s'adapter si elle souhaite répondre à cette pression extérieure et être en mesure d'augmenter le recrutement d'étudiants (E2). L'université devra apprendre à mieux articuler et justifier ses pratiques si elle souhaite se distinguer afin d'obtenir du financement.

Il sera nécessaire pour l'Université de Moncton de se pencher sur la conception Apprenante (E3). Cette conception est très présente dans les écoles. Les élèves qui accéderont à l'université auront des attentes auxquelles l'université devra s'ajuster (E6). Il faudra innover en pédagogie et créer des partenariats avec d'autres institutions. Il est nécessaire d'établir un équilibre entre la conception Académique et la conception Politique, tout en continuant à s'améliorer comme université (E3).

\section{Conclusion}

Nos objectifs de recherche étaient de: 1) vérifier l'application de la typologie de Larouche et al. (2012) dans le contexte d'une université francophone d'une province de l'Atlantique; 2) explorer auprès des dirigeants de cette université l'utilité de la typologie comme outil de gouvernance, de planification, d'évaluation ou de gestion.

Les dirigeants interrogés ont mentionné plusieurs réalités signifiantes illustrant les conceptions de la typologie à l'Université de Moncton. La diversité de leurs perceptions est illustrée et cette diversité se manifeste d'un secteur, d'une discipline ou d'une faculté à l'autre, selon les contextes et les dirigeants en place.

Les dirigeants ont également confirmé l'utilité de la typologie dans différents processus administratifs, comme outil de gouvernance et de planification, comme outil de réflexion pour élaborer une vision ou des orientations, comme outil de gestion dans les processus d'embauche, dans la prise de décision, pour obtenir des consensus et enfin comme outil d'évaluation multidimensionnel. 


\section{NOTES}

1 La typologie est décrite brièvement à la partie 2.

2 Ce projet de recherche s'inscrit dans la poursuite des travaux amorcés dans la recherche de Larouche (2011) qui a reçu le prix George-L-Geis pour la meilleure thèse de doctorat dans le domaine de l'enseignement supérieur au Canada.

3 Le lecteur intéressé par une description complète de la typologie peut se référer à Larouche et al. (2012), repéré à http://journals.sfu.ca/cjhe/index.php/cjhe/article/viewFile/2429/183771

4 Dans ce texte, le mot Université écrit avec une majuscule désigne l'institution générique de l’Université.

5 Le masculin est utilisé à titre d'épicène, il concerne autant les personnes de genre féminin que masculin.

6 Les administrateurs ont signé un formulaire de consentement écrit conformément au certificat éthique obtenu portant le numéro CE-602.409.03 à l’UQAC et reconnu par l’Université de Moncton no-1516-034.

7 Le détail des réalités signifiantes mentionnées est présenté à la partie 3.

8 Dans cette partie, les propos des dirigeants interrogés sont identifiés comme suit : E1, E2, E3, E4, E5, E6, E7 et E8.

9 Les exemples détaillés de son utilisation sont présentés à la partie 4.

\section{RÉFÉRENCES}

Amaral, A., Meek, V. L. et Larsen, I. M. (dir.) (2003). The higher education managerial revolution. Dordrecht, Pays-Bas: Kluwer Academic Publisher.

Axelrod, P. (2002). V alues in conflict: The university, the marketplace, and the trials of liberal education, Montreal, Québec: McGill-Queen's University Press.

Birnbaum, R. (1988). How colleges work. The cybernetics of academic organization and leadership. San Francisco, CA: Jossey-Bass.

Bruckmann, S. et Carvalho, T. (2014). The reform process of Portuguese higher education institutions: From collegial to managerial governance. Tertiary Education and Management, 20(3), 193-206.

Clark, B.R. (1998). Creating entrepreneurial universities: Organizational pathways of transformation. Oxford, Angleterre : Pergamon Press.

Dill, D. (2003). Le paradoxe de la qualité académique : Implications pour les universités et les politiques publiques. Revue des sciences de l'éducation. 29(2), 337-352.

Duke, C. (1992). The learning university: Toward a new paradigm? Buckingham, Angleterre: Society for research into Higher Education et Open University Press.

Fédération québécoise des professeures et professeurs d'université (FQPPU) (1997). L'Université comme service public. Communication présentée par le Comité exécutif de la FQPPU au Congrès de la FQPPU, Magog-Orford, Québec.

Freitag, M. (2002). L'université aujourd'hui : Les enjeux du maintien de sa mission institutionnelle d'orientation de la société. Dans G. Gagné (dir.), Main basse sur l'éducation. Montréal, Québec : Éditions Nota bene.

Garvin, D. (1993). Building a learning organization. Harvard Business Revue, 71(4), 78-84.

James, R. (2000). L'assurance qualité et le difficile problème de la gestion du savoir organisationnel en milieu universitaire. Gestion de l'enseignement supérieur, 12(3), 45-64.

Larouche, C. (2011). La validation d'une typologie des conceptions des universités en vue d'évaluer leur performance (Thèse de doctorat inédite). Université Laval.

Larouche, C., Savard, D. et Héon, L. (2016). Analyse typologique des plans stratégiques des universités québécoises Spécificité et alignement stratégique. Présentation aux États généraux de l'enseignement supérieur, Université Laval, Québec (mai, 2017).

Larouche, C., Savard, D., Héon, L. et Moisset, J.-J. (2012). Typologie des conceptions des universités en vue d'évaluer la performance : Rendre compte de la diversité pour en saisir la complexité. Revue canadienne d'enseignement supérieur. 42(3), $45-64$.

Larouche, C., Savard, D., Héon, L. et Moisset, J.-J. (2016). Analyse typologique des plans stratégiques des universités québécoises. Special issue: Governance in higher education. The Canadian Journal of Higher Education. 46(3), 18-40. 
Larouche, C., Savard, D. et Richard, J.-F. (2016). Applicabilité et utilité de la typologie de Larouche et al. (2012) comme outil de gouvernance, de planification et de gestion. Rapport de recherche. Document inédit.

Larouche, C., Savard, D. et Richard, J.-F. (2017). Applicabilité et utilité de la typologie de Larouche et al. (2012) comme outil de gouvernance, de planification et de gestion. Phase 2, Analyse du plan stratégique de l'Université de Moncton. Rapport de recherche. Document inédit.

Mead, P. (1995). Utilising the university as a learning organization to facilitate quality improvement. Quality in Higher education, 1(2), 111-121.

Ministère de l'Éducation (2000). Politique québécoise de financement des universités. Québec, Québec : Gouvernement du Québec.

Mintzberg, H., Ahlstrand, B. et Lampel, J. (1999). Safari en pays stratégie : l'exploration des grands courants de la pensée stratégique. Paris, France : Village Mondial.

Morgan, G. (1999). Images de l'organisation. Québec, Québec : Presses de l’Université Laval.

Morin, E. M., Savoie, A. et Beaudin, G. (1994). L'efficacité de l'organisation : Théories, représentations et mesures. Montréal, Québec : Gaëtan Morin.

Porter, M. E. (1993). L'avantage concurrentiel des nations. Paris, France : Inter-Éditions.

Quinn, R. E. et Rohrbaugh, J. (1983). A spatial model of effectiveness criteria: towards a competing values approach to organizational analysis. Management Science, 29(3), 363.

Ramsden, P. et Martin, E. (1996). Recognition of good university teaching: Policies from an Australian study. Studies in Higher Education, 16(2), 129-150. 


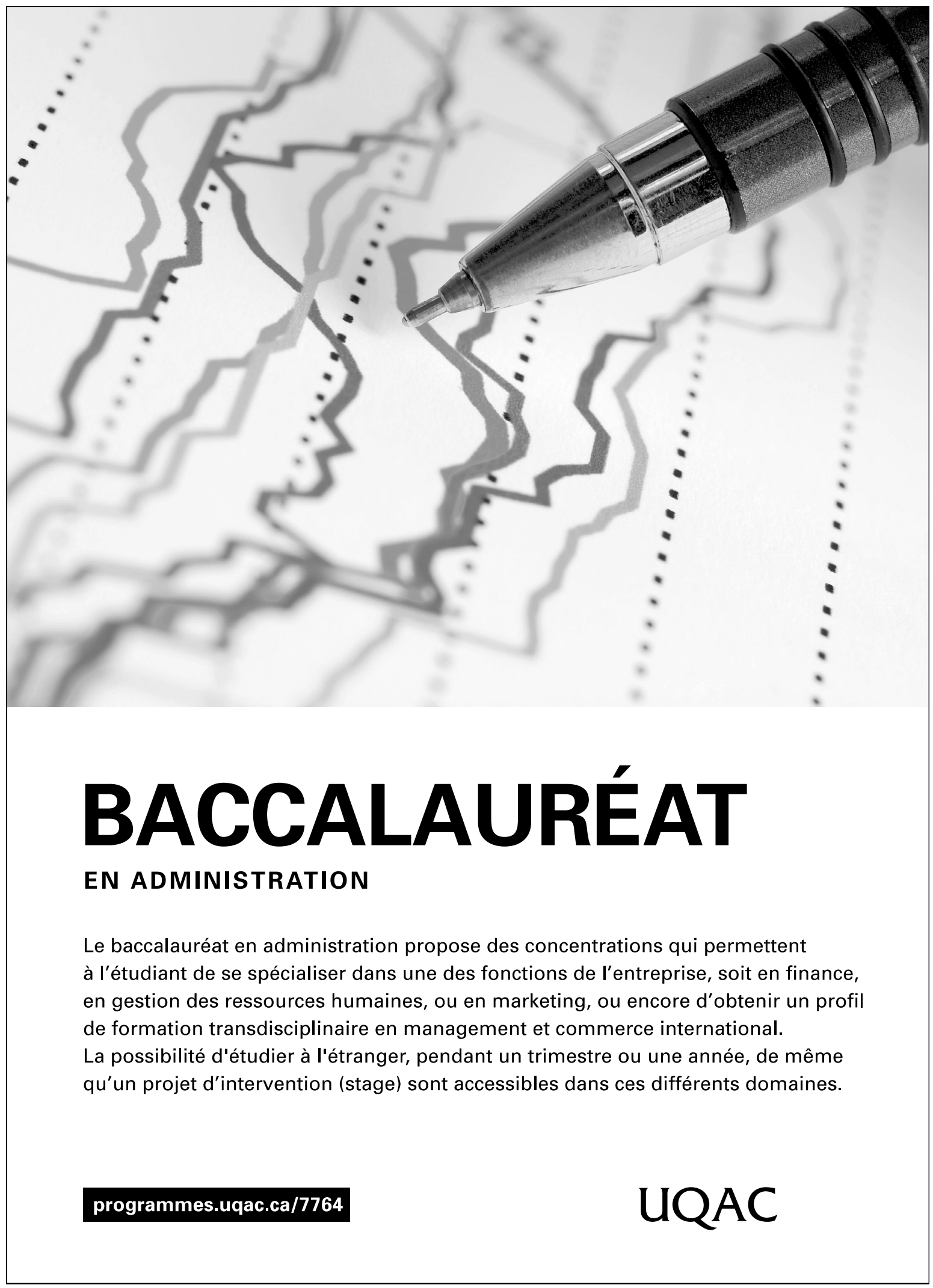

